Article

\title{
Digital-Control-Based Approximation of Optimal Wave Disturbances Attenuation for Nonlinear Offshore Platforms
}

\author{
Xiao-Fang Zhong ${ }^{1}$, Yu-Hong Sun ${ }^{1, *}$, Shi-Yuan Han ${ }^{2}$, Jin Zhou ${ }^{2}$ and Dong Wang ${ }^{2}$ \\ 1 School of Information Technology, Shandong Women's University, Jinan 250300, China; \\ swu_zhongxf@126.com \\ 2 Shandong Provincial Key Laboratory of Network Based Intelligent Computing, University of Jinan, \\ Jinan 250022, China; shiyuanhan@126.com (S.-Y.H.); ise_zhouj@ujn.edu.cn (J.Z.); \\ ise_wangd@ujn.edu.cn (D.W.) \\ * Correspondence: swu_sunyh@126.com; Tel.: +86-531-8276-6503
}

Received: 20 October 2017; Accepted: 22 November 2017; Published: 1 December 2017

\begin{abstract}
The irregular wave disturbance attenuation problem for jacket-type offshore platforms involving the nonlinear characteristics is studied. The main contribution is that a digital-control-based approximation of optimal wave disturbances attenuation controller (AOWDAC) is proposed based on iteration control theory, which consists of a feedback item of offshore state, a feedforward item of wave force and a nonlinear compensated component with iterative sequences. More specifically, by discussing the discrete model of nonlinear offshore platform subject to wave forces generated from the Joint North Sea Wave Project (JONSWAP) wave spectrum and linearized wave theory, the original wave disturbances attenuation problem is formulated as the nonlinear two-point-boundary-value (TPBV) problem. By introducing two vector sequences of system states and nonlinear compensated item, the solution of introduced nonlinear TPBV problem is obtained. Then, a numerical algorithm is designed to realize the feasibility of AOWDAC based on the deviation of performance index between the adjacent iteration processes. Finally, applied the proposed AOWDAC to a jacket-type offshore platform in Bohai Bay, the vibration amplitudes of the displacement and the velocity, and the required energy consumption can be reduced significantly.
\end{abstract}

Keywords: nonlinear offshore platform; digital control; nonlinear compensated component; disturbance attenuation; irregular wave force

\section{Introduction}

The offshore platforms provide the basic support for marine operation, such as the oil exploration, drilling operations and transportation. A considerable amount of theoretical and experimental research effort has been aimed at improving the control performance of offshore platforms, including passive and active control [1,2]. Passive control is the classic technology to enhance the safety by using excessive construction materials to guarantee the stability of the offshore structures. Nevertheless, passive systems have limitations in improving control performance, even with the huge energy cost. In comparison, active control technologies have great potential to meet the optimal performance requirements with low consumption requirement [3]. Recently, various kinds of active schemes have been derived and employed for offshore platforms, e.g., using the concepts of non-fragile control [4], sliding mode theory [5] and $H \infty$ control [6].

With the development of advanced sensor, actuator and digital control technology, offshore platforms under digital control system have been attracting extensive attention in the field of controller design $[7,8]$. Offshore platforms generally involve sophistication of the superstructure in the deep water, 
in which various inevitable factors directly affect offshore platforms in ocean environment, especially vibration caused by external wave force [9]. In order to describe and identify the characteristic of wave force, various idealized spectra are employed to describe the empirical relationship that defines the distribution of energy with frequency within the ocean [10], such as the Pierson-Moskowitz spectrum [11] and Joint North Sea Wave Project (JONSWAP) spectrum [12]. Compared to the Pierson-Moskowitz spectrum, the JONSWAP spectrum is effectively a fetch-limited version to analyze the wave force in the wave-frequency domain [13]. Many control schemes were proposed to attenuate the wave-induced vibration thereby ensuring the safety and satisfying the performance requirements. For example, the wave vibration problem was formulated as an optimal tracking control problem and a corresponding tracking control scheme was proposed to reduce the displacement and velocity of offshore platform in [14]; a network-based state feedback control scheme was designed for steel offshore platform based on the stability criterion in [15] with small control consumption; a non-fragile sampled-data controller was proposed in [4], thereby reducing the oscillation amplitudes of the offshore platform; an event-triggered controller was derived from the Lyapunov-Krasovskii function approach to guarantee the stability of offshore structure in [16]. It should be noted that the aforementioned works are with huge energy consumption. Meanwhile, the optimal vibration control theories provide efficient methods to improve the control performance of control systems with smaller control consumption. Therefore, one target of this paper is to apply the optimal control technology to the offshore platform under digital control system.

In general, offshore platforms have their inevitable nonlinear characteristics caused by rigid structure, multiplying springs and dampers, flexibility and complexity structures. Unfortunately, the unsafely behaviors and destabilization of offshore platforms mainly result from nonlinear dynamics. Many researchers have engaged in the efforts to the implementation of control schemes for nonlinear offshore platforms, e.g., a robust mixed control method for wave-excited offshore jacket platforms is proposed in [17] to minimize the upper bound of the performance measure on platform dynamics satisfying some norm bound constraint simultaneously; a novel sliding mode control scheme is proposed by using information about mixed current and delayed states in [18]; by combining a sliding mode control technique, the adaptive control algorithm and wavelet support vector machine, an adaptive integral sliding mode control to handle the nonlinear behavior of the offshore platform in [19]. Meanwhile, the optimal vibration control theories provide efficient methods to improve the control performance of control systems with smaller control consumption [20-23]. However, optimal control for a nonlinear discrete system will lead to a Hamilton-Jacobi-Bellman (HJB) equation with no exact analytical solution except [24]. In order to obtaining approximate solutions to the HJB equation, many approaches have been developed, such as the power series approximation [25], the successive Galerkin approach [26], and approximating sequence of Riccati equations approach [27]. However, it still is difficult to seek the solution under the external disturbance and nonlinear dynamics. This is the motivation of this paper.

The irregular wave disturbance attenuation problem for a nonlinear offshore platform under digital control system is investigated in this paper. An approximation of optimal wave disturbances attenuation controller (AOWDAC) is proposed to compensate the external irregular wave force and nonlinear dynamics. First, the discrete nonlinear jacket-type offshore platform is established under a digital control system, in which the external wave force acting on the offshore platform is viewed as the output of an external system. In order to minimize the average quadratic performance index and energy consumption, AOWDAC is developed to obtain the approximation solution of a derived nonlinear two-point-boundary-value (TPBV) problem caused from the nonlinear offshore structure, which is made up of disturbance compensation of wave force, feedback items of offshore platforms and the vector sequences of nonlinear compensation. Based on the value of performance index in the process of iteration, the feasibility of AOWDAC is realized. By analyzing the displacement and velocity of a jacket-type offshore platform located in Bohai Bay, the effectiveness of developed AOWDAC is proved compared with an optimal feedback and feedfoward vibration controller (OFFVC). 
The rest of this paper is as follows. The irregular wave attenuation problem for a nonlinear offshore platform under a digital control system is formulated in Section 2. The main results are shown in Section 3, in which the AOWDAC is designed to compensate the irregular wave force and nonlinear dynamics, and the feasibility of AOWDAC is realized by using the designed iterative algorithm. In Section 4, a jacket-type platform located in Bohai Bay is introduced to prove the effective of the designed AOWDAC and algorithm. Concluding remarks are drawn in Section 5.

\section{Problem Formulation}

In this section, the model of a jacket-type offshore platform under a digital control system is introduced first, and the wave force derives from the output of an external system. Then, the optimal wave disturbances attenuation problem is formulated with respect to a quadratic performance index.

\subsection{Jacket-Type Offshore Platform}

The simple structure of jacket-type offshore platform with an active mass damper (AMD) device is shown in Figure 1 [28]. The global dynamic equation of a jacket-type offshore platform is given by the following coupled equations:

$$
\left\{\begin{array}{c}
m_{1} \ddot{x}_{1}(t)=\left(m_{1} \omega_{1}^{2}+m_{2} \omega_{2}^{2}\right) x_{1}(t)-2\left(\xi_{1} m_{1} \omega_{1}+\xi_{2} m_{2} \omega_{2}\right) \dot{x}_{1}(t) \\
+\omega_{2}^{2} m_{2} x_{2}(t)+2 \xi_{2} m_{2} \dot{x}_{2}(t)+p(t)-\alpha_{1} x_{1}^{3}(t)-\alpha_{3} x_{1}^{5}(t)-u(t), \\
m_{2} \ddot{x}_{2}(t)=m_{2} \omega_{2}^{2}\left(x_{1}(t)-x_{2}(t)\right)+2 \xi_{2} m_{2} \omega_{2}\left(\dot{x}_{1}(t)-\dot{x}_{2}(t)\right)+u(t),
\end{array}\right.
$$

where $m_{1}, \omega_{1}$ and $\xi_{1}$ denote the modal mass, frequency, and damping ratio of jacket-type platform, respectively; $m_{2}, \omega_{2}$ and $\xi_{2}$ are the mass, frequency, and damping ratio of the AMD; $p(t)$ and $u(t)$ represent the irregular wave force and control force. $x_{1}$ is the corresponding modal coordinate that refers to the deck motion of a jacket-type offshore structure; $x_{2}$ is the displacement of the AMD.

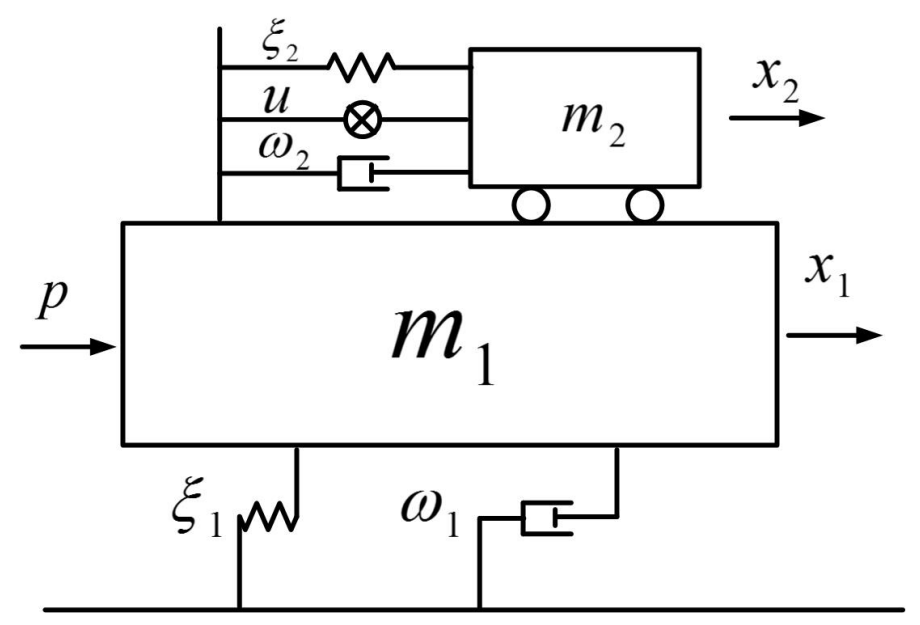

Figure 1. The simple structure of jacket-type offshore platform with an active mass damper device.

By defining $\bar{x}(t)=\left[\begin{array}{llll}x_{1}(t) & x_{2}(t) & \dot{x}_{1}(t) & \dot{x}_{2}(t)\end{array}\right]^{T}$, the state-space model of the jacket platform can be described as

$$
\dot{\bar{x}}(t)=\bar{A} \bar{x}(t)+\bar{B} u(t)+\bar{D} p(t)+\bar{f}(\bar{x}(t)), \quad \bar{x}(0)=\bar{x}_{0}
$$


where

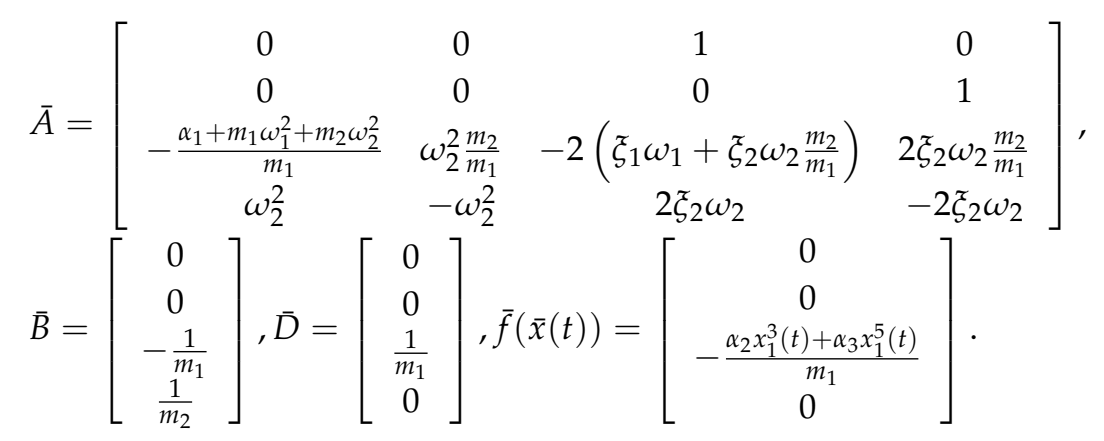

Defining the sampling period as $T$, the discrete-time model of jacket platform (2) under a digital control system can be described as

$$
x(k+1)=A x(k)+B u(k)+D p(k)+f(x(k)), \quad \bar{x}(0)=\bar{x}_{0},
$$

where $A=e^{\bar{A} T}, B=\int_{0}^{T} e^{\bar{A} T} \bar{B} d t, D=\int_{0}^{T} e^{\bar{A} T} \bar{D} d t$, and $f(x(k))=\int_{0}^{T} e^{\bar{A} T} \bar{f}(\bar{x}(t)) d t$.

\subsection{Irregular Wave Disturbances}

Based on the linear wave theory and the JONSWAP wave spectrum, the acceleration $\alpha(z, t)$ and horizontal velocity $v(z, t)$ of the ocean water particle can be described as

$$
\alpha(z, t)=-\omega^{2} \frac{\cosh (n z)}{\sinh (n d)} \tan (\omega t) \eta(t), \quad v(z, t)=\omega \frac{\cosh (n z)}{\sinh (n d)} \tan (\omega t) \eta(t),
$$

where $z$ is the vertical coordinate axis, $d$ denotes the ocean depth, $n$ is the wave number, and $\eta(t)$ denotes the wave elevation. Then, the wave force $p(z, t)$ acting on the column of jacket-type platform can be described as

$$
p(z, t)=K_{d} \sqrt{\frac{8}{\pi}}\left[\int_{0}^{\omega}\left|\omega \frac{\operatorname{conh}(n \varsigma)}{\sinh (n d)} \tan (\omega t)\right|^{2} S_{\eta}(\omega) d \omega\right]^{\frac{1}{2}} v(z, t)+K_{m} \alpha(z, t),
$$

where $S_{\eta}(\omega)$ is the irregular wave condition, $K_{d}=(1 / 2) C_{d} \rho d_{e}$ and $K_{m}=(1 / 4) C_{m} \rho \pi\left(d_{e}\right)^{2}$, in which $\rho$ is the density of ocean fluid, $d_{e}$ is the characteristic diameter of the jacket-type platform legs, $C_{d}$ and $C_{m}$ are the drag and inertia parameters, respectively.

The total wave force $p(t)$ acting on the jacket-type offshore platform can be calculated from

$$
\begin{aligned}
p(t) & =\int_{0}^{d} p(z, t) \varphi(z) d z \\
& =\left\{\int _ { 0 } ^ { d } \left[K_{d} \sqrt{\frac{8}{\pi}}\left[\int_{0}^{\omega}\left|\omega \frac{\cosh (n z)}{\sinh (n d)} \tan (\omega t)\right|^{2} S_{\eta}(\omega) d \omega\right]^{\frac{1}{2}} \omega \frac{\cosh (n z)}{\sinh (n d)} \tan (\omega t)\right.\right. \\
& \left.\left.-K_{m} \omega^{2} \frac{\cosh (n z)}{\sinh (n d)} \tan (\omega t)\right] \varphi(z) d z\right\} \eta(t) \\
& =T(\omega) \eta(t) .
\end{aligned}
$$

Letting $\bar{V}_{j}=A_{j} \cos \left(-\omega_{j} t+\varepsilon_{j}\right)$ and $\bar{v}(t)=\left[\begin{array}{lll}\bar{v}_{1}(t) & \cdots & \bar{v}_{r}(t)\end{array}\right]^{T}$, we have

$$
\ddot{v}(t)=\left[\begin{array}{ccc}
-\omega_{1}^{2} & 0 & 0 \\
0 & \ddots & 0 \\
0 & 0 & -\omega_{r}^{2}
\end{array}\right]=G_{a} \bar{v}(t)
$$


where $\varepsilon_{j}$ denotes random phase angles in the range of $\left[\begin{array}{ll}0 & \pi\end{array}\right]$.

Introducing $w(t)=\left[\begin{array}{cc}\bar{v}(t) & \dot{v}(t)\end{array}\right]^{T}$ and noting $\eta(t)=\left[\begin{array}{ccc}1 & \cdots & 1\end{array}\right] \bar{v}(t)$, the following external system can be designed to describe the wave force $p(t)$, which is described as

$$
\left\{\begin{array}{c}
\dot{w}(t)=\left[\begin{array}{cc}
0 & I \\
G_{a} & 0
\end{array}\right] w(t)=G_{c} w(t) \\
f(t)=T(\omega)\left[\begin{array}{lll}
1 & \cdots & 1
\end{array}\right] \bar{v}(t)=T(\omega)\left[\begin{array}{lll}
1 & \cdots & 1
\end{array}\right]\left[\begin{array}{ll}
I & 0
\end{array}\right] w(t)=F w(t) .
\end{array}\right.
$$

Under the sampling period $T$, the discrete-time form of wave force (9) under digital control system can be described as

$$
\left\{\begin{array}{l}
w(k+1)=G w(k), \\
p(k)=F_{c} w(k)
\end{array}\right.
$$

where $G=e^{G_{c} T}$. All of the eigenvalues $\lambda_{i}(G)$ of $G$ satisfy

$$
\left|\lambda_{i}(G)\right|=1, \quad i=1,2, \ldots, 2 r .
$$

It is assumed that $(F, G)$ is completely observable.

\subsection{Discrete Optimal Irregular Wave Disturbances Attenuation Problem}

Due to the wave force $p(k)$ and nonlinear item $f(x(k))$, the system state $x(k)$ and controller $u(k)$ cannot converge to zero jointly. Therefore, the classic quadratic performance index is not applied to this wave disturbance attenuation problem. Then, the following average quadratic performance index is chosen as

$$
J=\lim _{N \rightarrow \infty} \frac{1}{2 N} \sum_{k=0}^{N}\left[y_{1}^{T}(k) Q y_{1}(k)+u^{T}(k) R u(k)\right],
$$

where $Q=C_{1}{ }^{T} Q C_{1} \in \mathbb{R}^{l \times l}$ is a positive semi-definite matrix and $R>0 \in \mathbb{R}$ is a constant.

Therefore, the optimal irregular wave disturbances attenuation problem can be formulated as designing the digital-control-based AOWDAC $u^{*}(k)$ for a nonlinear offshore platform (2) and wave forces (9) so that the average quadratic performance index (12) can be obtained the minimum value.

In order to design the digital-control-based AOWDAC, we introduce the following lemmas.

Lemma 1. Let $A_{1} \in \mathbb{R}^{n \times n}, B_{1} \in \mathbb{R}^{m \times m}$, and $X \in \mathbb{R}^{n \times m}$. The matrix equation

$$
A_{1} X B_{1}-X=C_{1}
$$

has a unique solution $X$ if and only if

$$
\lambda_{i}\left(A_{1}\right) \times \lambda_{j}\left(B_{1}\right) \neq 1, \quad i=1, \ldots, n, \quad j=1, \ldots, m,
$$

where $\lambda(\cdot)$ denotes eigenvalues of matrix [28].

Lemma 2. The discrete nonlinear system is expressed as

$$
\left\{\begin{array}{l}
z(k+1)=H(k) z(k)+h(z(k)) \\
z(0)=\varphi(0)
\end{array}\right.
$$


where $z(k)$ is the system state, $\varphi(0)$ denotes the initial state vale, and $h(z(k))$ satisfies the Lipschitz condition. The following sequence $z^{(j)}$ converges to the solution of discrete system (15), which is described as

$$
\left\{\begin{array}{l}
z^{(0)}(k)=\prod_{m=1}^{k} H(k-m) \varphi(0), \quad k=1,2, \ldots \\
z^{(j)}(k)=\prod_{m=1}^{k} H(k-m) \varphi(0)+\prod_{i=0}^{k-1}\left\{\left[\prod_{m=1}^{k} H(k-m)\right] h\left(z^{(j-1)}(k)\right)\right\} \\
z^{(j)}(0)=\varphi(0), \quad k=1,2, \ldots ; \quad j=1,2, \ldots,
\end{array}\right.
$$

where $\prod_{m=1}^{k} H(k-m)=I, I$ is the unit matrix [27].

\section{Main Results}

In this section, the digital-control-based AOWDAC will be designed, which includes the feedback item, feedforward item and the nonlinear compensation item. After that, the implement ability of digital-control-based AOWDAC will be satisfied by using the designed numerical algorithm.

\subsection{Design of AOWDAC}

Theorem 1. Considering the wave disturbance attenuation problem for a discrete jacket-type platform (4) and wave force (10) with respective to the average quadratic performance index (12), the digital-control-based AOWDAC can be designed as

$$
u^{*}(k)=-R^{-1} B^{T}\left(A^{T}+f_{x}^{T}(x(k))\right)^{-1}\left[\left(P_{1}-Q\right) x(k)+P_{2} w(k)+\lim _{j \rightarrow \infty} g^{(j)}(k+1)\right],
$$

where the item with $x(k)$ denotes the feedback component; the item with $w(k)$ is the feedforward component; the item with $g^{(j)}(k+1)$ denotes the nonlinear compensation; $f_{x}(x(k))$ denotes a Jacobian matrix of $f(x(k))$ with respect to $x(k) ; P_{1}$ is the unique positive-semi-definite solution of the following Riccati matrix equation

$$
P_{1}=Q+A^{T} P_{1} S^{-1} A
$$

with $S=I+B_{1} R^{-1} B_{1}^{T} P_{1} ; P_{2}$ is the unique solution of the following Stein matrix equation

$$
P_{2}=A^{T} P_{1} G+A^{T} P_{1} S^{-1}\left(D F-B_{1} R^{-1} B_{1}^{T} P_{2} G\right) .
$$

The sequence of $\left\{g^{(i)}(k)\right\}$ can be calculated from

$$
\left\{\begin{array}{l}
g^{(j)}(\infty)=0, \quad j=0,1, \cdots \\
g^{(0)}(\infty)=0, \quad j=0,1, \cdots, \\
g^{(j)}(k)=\left(A^{T}+f_{x}^{T}\left(x^{(j-1)}(k)\right)\right)\left\{P _ { 1 } S ^ { - 1 } \left[f\left(x^{(j-1)}(k)\right)\right.\right. \\
\left.\left.\quad-B R^{-1} B^{T} g^{(j)}(k+1)\right]+g^{(j)}(k+1)\right\}+f_{x}^{T}\left(x^{(j-1)}(k)\right) \times \\
\left\{P_{1} S^{-1}\left[A x^{(j-1)}+\left(D F-B R^{-1} B^{T} P_{2} G\right) w(k)\right]+P_{2} G w(k)\right\} \\
j=0,1, \cdots ; \quad k=0,1, \cdots
\end{array}\right.
$$

where the sequence of $\left\{x^{(j)}(k)\right\}$ in (20) can be obtained from

$$
\left\{\begin{aligned}
& x^{(j)}(0)=x_{0}, \quad j=0,1, \cdots \\
& x^{(j)}(k)=S^{-1}\left[A x^{(j)}(k)+\left(D F-B R^{-1} B^{T} P_{2} G\right) w(k)-\right. \\
&\left.-B R^{-1} B^{T} g^{(j)}(k+1)+f\left(x^{(j-1)}(k)\right)\right] \\
& j=0,1, \cdots ; \quad k=0,1, \cdots .
\end{aligned}\right.
$$


Proof of Theorem 1. Applying the optimal control theory to the following Hamilton function

$$
\begin{aligned}
H(\cdot)= & \frac{1}{2}\left(x^{T}(k) Q x(k)+u^{T}(k) R u(k)\right)+ \\
& \lambda^{T}(k+1)(A x(k)+B u(k)+D p(k)+f(x(k))) .
\end{aligned}
$$

Then, we have the following TPBV problem with nonlinear items

$$
\left\{\begin{array}{l}
x(k+1)=A x(k)-B R^{-1} B^{T} \lambda(k+1)+D p(k)+f(x(k)), \quad x(0)=x_{0}, \\
\lambda(k)=Q x(k)+\left(A^{T}+f_{x}^{T}(x(k))\right)^{-1} \lambda(k+1), \quad \lambda(\infty)=0 .
\end{array}\right.
$$

Meanwhile, the optimal control law can be described as

$$
u(k)=-R^{-1} B^{T} \lambda(k+1) .
$$

In order to solve the above TPBV problem, $\lambda(k)$ is designed as

$$
\lambda(k)=P_{1} x(k)+P_{2} w(k)+g(k) .
$$

Then, one gets

$$
\left\{\begin{array}{l}
x(k+1)=S^{-1}\left\{A x(k)+\left(D F-B R^{-1} B^{T} P_{2} G\right) w(k)-B R^{-1} B^{T} g(k+1)+f(x(k))\right\} \\
\lambda(k+1)=\left(A^{T}+f_{x}^{T}(x(k))\right)^{-1}\left(\left(P_{1}-Q\right) x(k)+P_{2} w(k)+g(k+1)\right) .
\end{array}\right.
$$

Substituting the second formula of (26) into (24), the optimal control law can be rewritten as

$$
u^{*}(k)=-R^{-1} B^{T}\left(A^{T}+f_{x}^{T}(x(k))\right)^{-1}\left[\left(P_{1}-Q\right) x(k)+P_{2} w(k)+g(k+1)\right] .
$$

Rearranging the second formula of (26), we have

$$
\begin{aligned}
\lambda(k)= & Q x(k)+\left(A^{T}+f_{x}^{T}(x(k))\right)^{-1} \lambda(k+1) \\
= & \left(Q+A^{T} P_{1} S^{-1} A\right) x(k)+\left(A^{T} P_{1} G+A^{T} P_{1} S^{-1}\left(D F-B R^{-1} B^{T} P_{2} G\right)\right) w(k) \\
= & \left(A^{T}+f_{x}^{T}(x(k))\right)\left\{P_{1} S^{-1}\left[f(x(k))-B R^{-1} B^{T} g(k+1)\right]+g(k+1)\right\}+ \\
& \left.f_{x}^{T}(x(k))\right)\left\{P_{1} S^{-1}\left(A x(k)+\left(D F-B R^{-1} B^{T} P_{2} G\right) w(k)\right)+P_{2} G w(k)\right\} .
\end{aligned}
$$

Noting the parameters of (25) and (28), we can obtain the Riccati Equation (18) and Stein Equation (19). Because the pair $(A, B)$ is controllable, it can be claimed that $P_{1}$ is the unique positive-semi-definite solution of (28). Noting (11) and the first formula (26), we have that all of the eigenvalues of $\left(A^{T} P_{1} S^{-1} B R^{-1} B^{T}\right)$ satisfy $\left|\lambda_{i}\left(A^{T} P_{1} S^{-1} B R^{-1} B^{T}\right)\right|<1$. Therefore, $P_{2}$ is the unique solution of the Stein matrix (19).

Meanwhile, the following TPBV problem can be derived from the first formula of (26) and (28):

$$
\left\{\begin{array}{c}
g(k)=\left(A^{T}+f_{x}^{T}(x(k))\right)\left\{P_{1} S^{-1}\left[f(x(k))-B R^{-1} B^{T} g(k+1)\right]+g(k+1)\right\}+ \\
f_{x}^{T}(x(k))\left\{P_{1} S^{-1}\left(A x(k)+\left(D F-B R^{-1} B^{T} P_{2} G\right) w(k)\right)+P_{2} G w(k)\right\} \\
x(k+1)=S^{-1}\left\{A x(k)+\left(D F-B R^{-1} B^{T} P_{2} G\right) w(k)-B R^{-1} B^{T} g(k+1)+f(x(k))\right\} \\
x(0)=x_{0}, \quad g(\infty)=0 .
\end{array}\right.
$$

Due to the nonlinear item $f(x(k))$, the analytical solution of (29) is difficult to be obtained. Based on Lemma 1, the vector sequences of $\left\{g^{(i)}\right\}$ and $\left\{x^{(i)}(k)\right\}$ are introduced to solve the TPBV problem (29). From Lemma 1, one gets

$$
\lim _{i \rightarrow \infty} g^{(i)}(k)=g(k), \quad \lim _{i \rightarrow \infty} x^{(i)}(k)=x(k) .
$$


Rearranging (20), (21) and (29), control sequence $\left\{u^{(i)}(k)\right\}$ can be described as

$$
u^{(i)}(k)=-R^{-1} B^{T}\left(A^{T}+f_{x}^{T}\left(x^{(i-1)}(k)\right)\right)^{-1}\left[\left(P_{1}-Q\right) x^{(i-1)}(k)+P_{2} w(k)+g^{(i-1)}(k+1)\right] .
$$

Rearranging the above control sequence $\left\{u^{(i)}(k)\right\}$, one gets

$$
\begin{aligned}
& u^{*}(k)=\lim _{i \rightarrow \infty} u^{(i)}(k)=-R^{-1} B^{T}\left(A^{T}+f_{x}^{T}\left(x^{(\infty)}(k)\right)\right)^{-1} \times \\
& {\left[\left(P_{1}-Q\right) x^{(\infty)}(k)+P_{2} w(k)+g^{(\infty)}(k+1)\right] . }
\end{aligned}
$$

From (32), the digital-control-based AOWDAC (17) can be formulated. The proof is completed.

\subsection{The Feasibility of AOWDAC}

The unfeasibility of digital-control-based AOWDAC (17) results from the term of $\lim _{j \rightarrow \infty} g^{(j)}(k+1)$, and the iteration number must be limited. Therefore, this subsection will design a numerical algorithm to decide the iteration number based on the performance index in each iteration.

Based on (17), the feasible controller is designed as

$$
u^{(M)}(k)=-R^{-1} B^{T}\left(A^{T}+f_{x}^{T}\left(x^{(M)}(k)\right)\right)^{-1}\left[\left(P_{1}-Q\right) x^{(M)}(k)+P_{2} w(k)+g^{(M)}(k+1)\right],
$$

where $M$ is determined from the following algorithm.

Step 1: Obtaining $P_{1}$ and $P_{2}$ from (18) and (19), defining $j=M=1$, the sequences $g^{(0)}(k)=g^{(1)}(k)=0$ and $x^{(\infty)}(0)=x_{0}$, and giving a small positive real constant $\varepsilon$;

Step 2: Setting $M=j$, and obtaining $g^{(j)}(k)$ from the third formula of (20);

Step 3: Calculating $u^{(M)}(k)$ from (33) and the state sequence $x^{(}(j)(k)$ from the second formula of (21);

Step 4: Calculating the performance index $J_{M}$ in the $M$ th iteration from

$$
J_{M}=\lim _{N \rightarrow \infty} \frac{1}{2 N} \sum_{k=0}^{N}\left(x^{(M)}(k)^{T} Q x^{M}(k)+u^{(M)}(k)^{T} Q u^{M}(k)\right) .
$$

Step 5: If $\left|\left(J_{M}-J_{M-1}\right) / J_{M}\right|<\varepsilon$, then stop and obtain the controller $u^{(M)}(k)$, else go to Step 6;

Step 6: Let $j=j+1$, return to Step 2.

\section{Simulation Results}

In this section, the parameters of offshore platform in Bohai Bay are given first. Then, the wave disturbance attenuation abilities of proposed digital-control-based AOWDAC are analyzed.

\subsection{Parameters of Wave Disturbance Attenuation Problem}

Taking the jacket-type offshore platform located in Bohai Bay into consideration, the parameters of installed AMD and offshore structure are listed in Table 1 [28].

Then, the matrices in (4) can be obtained under sampling period $\mathrm{T}=0.1 \mathrm{~s}$ as follows:

$$
A=\left[\begin{array}{cccc}
0.979 & 0.000 & 0.099 & 0.000 \\
0.021 & 0.979 & 0.005 & 0.095 \\
-0.418 & 0.004 & 0.970 & 0.001 \\
0.395 & -0.415 & 0.100 & 0.899
\end{array}\right], B=\left[\begin{array}{c}
-0.001 \\
0.062 \\
-0.012 \\
1.221
\end{array}\right], D=\left[\begin{array}{l}
0.001 \\
0.000 \\
0.013 \\
0.001
\end{array}\right] \text {. }
$$


Table 1. Parameters of jacket-type offshore structure with an active mass damper device.

\begin{tabular}{cccc}
\hline Name of Parameter & Variable & Value & Unit \\
\hline Mass of AMD & $m_{2}$ & 11,855 & $\mathrm{~kg}$ \\
Nature frequency of AMD & $\omega_{2}$ & 2.33 & $\mathrm{rad} / \mathrm{s}$ \\
Structure damping ratio of AMD & $\xi_{2}$ & 9.32 & $\%$ \\
First modal mass of offshore structure & $m_{1}$ & $2,371,100$ & $\mathrm{~kg}$ \\
Nature frequency & $\omega_{1}$ & 2.20 & $\mathrm{rad} / \mathrm{s}$ \\
Structure damping ratio & $\xi_{1}$ & 4 & $\%$ \\
Shape function of first mode & $\varphi(z)$ & $1-\cos (\pi z / 2 L)$ & $/$ \\
Equivalent characteristic diameter of legs & $d_{3}$ & 1.7 & $\mathrm{~m}$ \\
Nonlinear parameter & $\alpha_{1}$ & $1,500,534$ & $/$ \\
Nonlinear parameter & $\alpha_{2}$ & $2,371,100$ & $/$ \\
Nonlinear parameter & $\alpha_{3}$ & $2,382,955$ & $/$ \\
\hline
\end{tabular}

Meanwhile, the parameters of irregular wave force are given in Table 2. Then, the irregular wave force $p(k)$ acting on the jacket-type offshore platform (35) can be calculated from (10) and displayed in Figure 2. The weight matrices in performance index (12) are chosen as $Q=\operatorname{diag}\{100,0,100\}$, and $R=1$.

Table 2. Parameters of irregular wave force.

\begin{tabular}{cccc}
\hline Name of Parameter & Variable & Value & Unit \\
\hline Water depth & $d$ & 13.2 & $\mathrm{~m}$ \\
Drag coefficient & $C_{d}$ & 1.2 & $/$ \\
Inertial coefficient & $C_{m}$ & 2.0 & $/$ \\
Density of ocean fluid & $\rho$ & 0.09 & $/$ \\
\hline
\end{tabular}

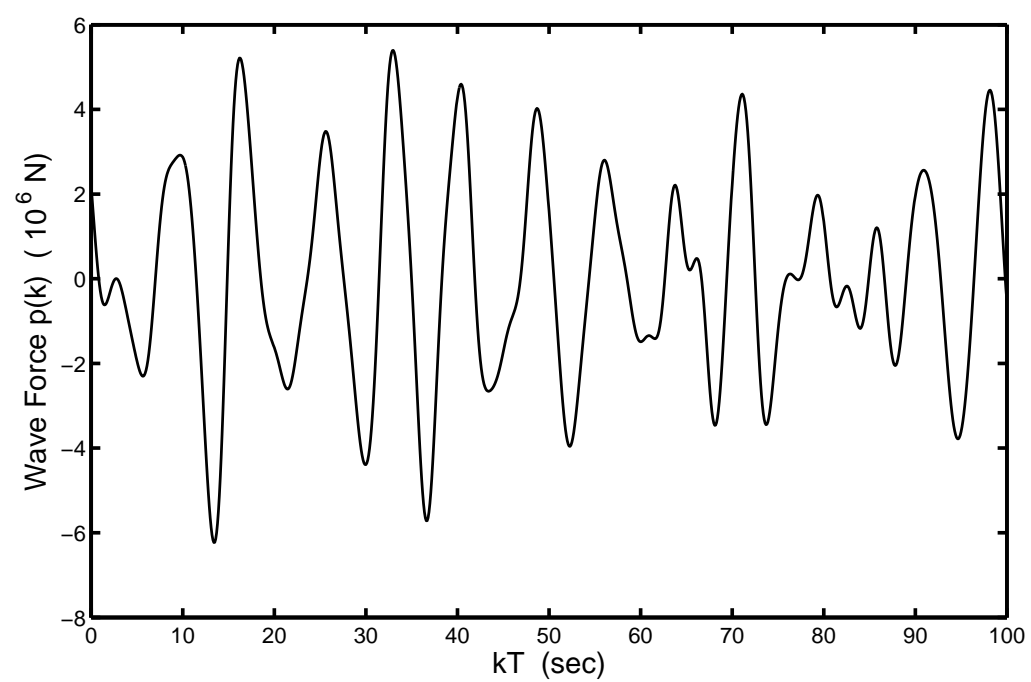

Figure 2. Irregular wave force acting on the jacket-type offshore platform.

\subsection{Analysis of Wave Disturbance Attenuation Abilities of Proposed AOWDAC}

In this subsection, in order to illustrate the effectiveness of proposed digital-control-based AOWDAC, the following optimal feedback and feedfoward vibration controller (OFFVC) designed in [28] is used for comparison purposes, which is described as

$$
u^{*}(k)=-\left(R+B^{T} P_{1} B\right)^{-1} B^{T}\left\{P_{1} A x(k)+P_{1} D p(k)+P_{2} G w(k)\right\} .
$$


Setting $\varepsilon=0.1$, the digital-control-based AOWDAC is obtained after iterative number $K=4$. The control performances $J$ in each iteration are shown in Table 3. Considering the offshore platform (4) with open-loop case (without control input), the proposed digital-control-based AOWDAC (33) and OFFVC (36), the curves of velocity, displacement and energy consumption of offshore platforms are displayed in Figures 3-5, respectively.

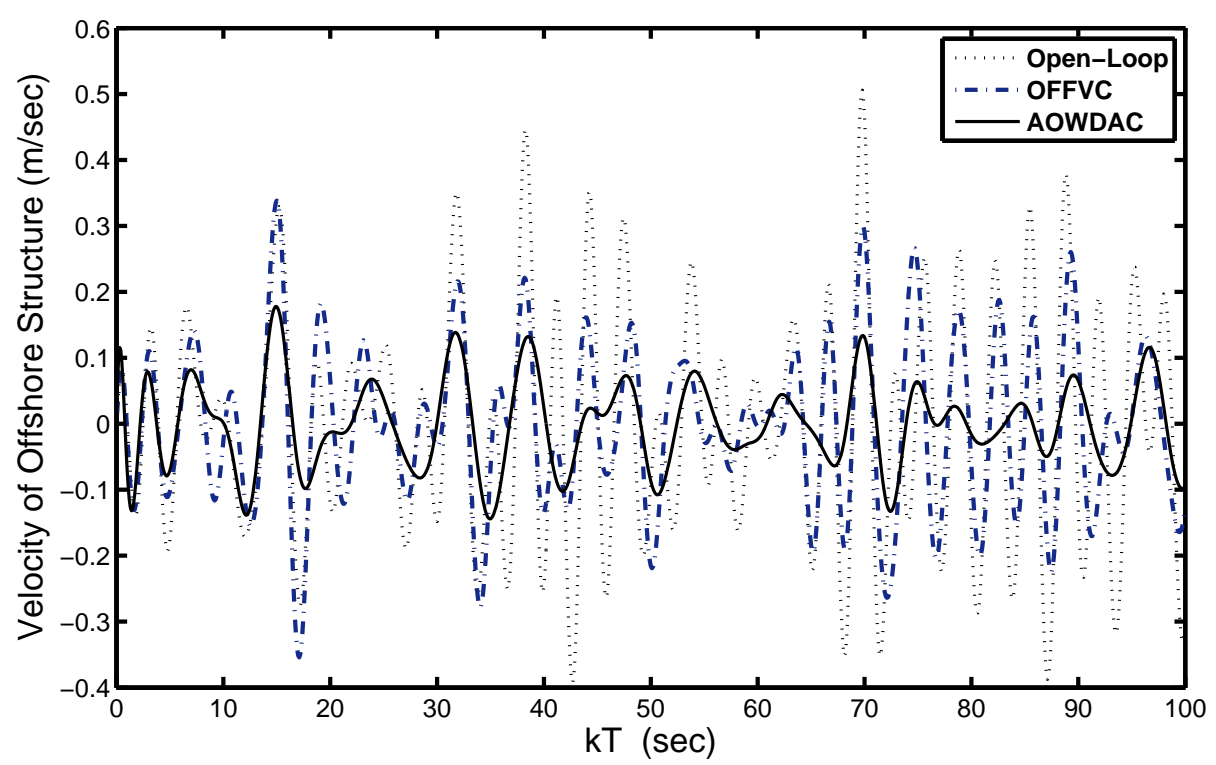

Figure 3. The curves of velocities of offshore platforms under different control schemes.

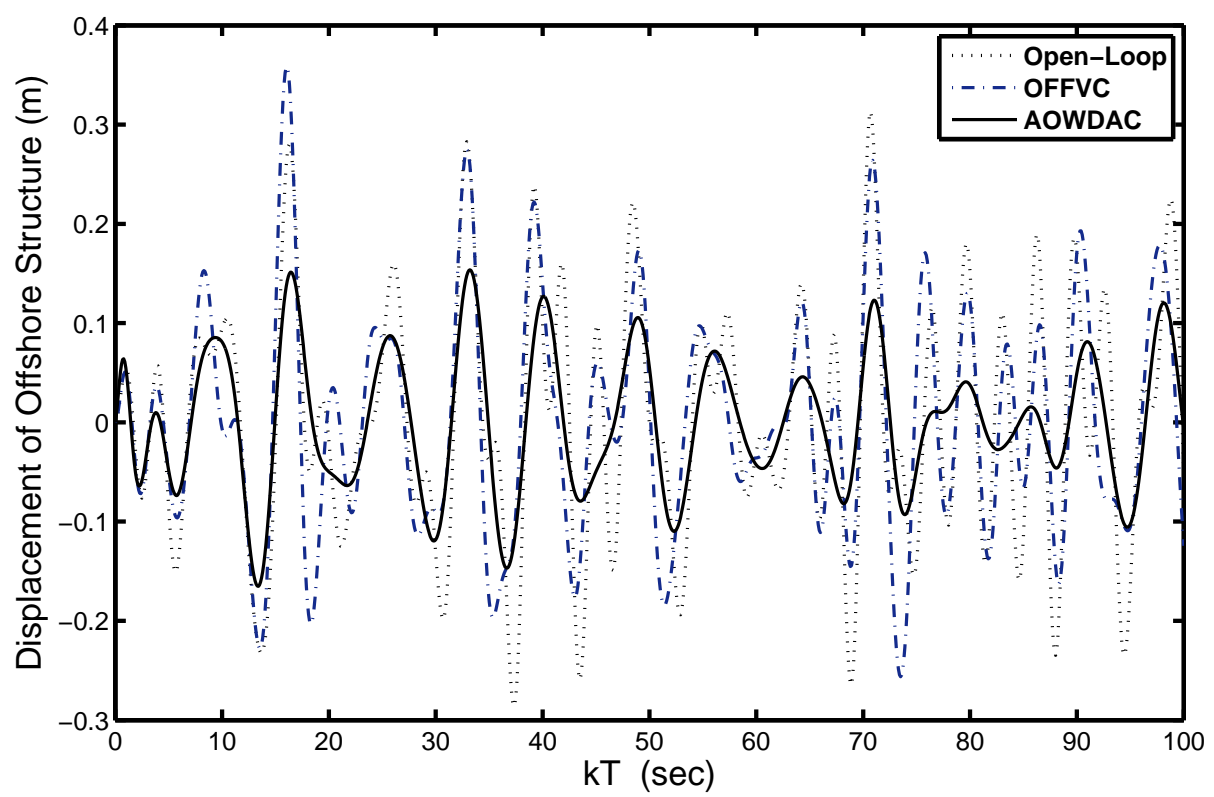

Figure 4. The curves of displacements of offshore platforms under different control schemes. 


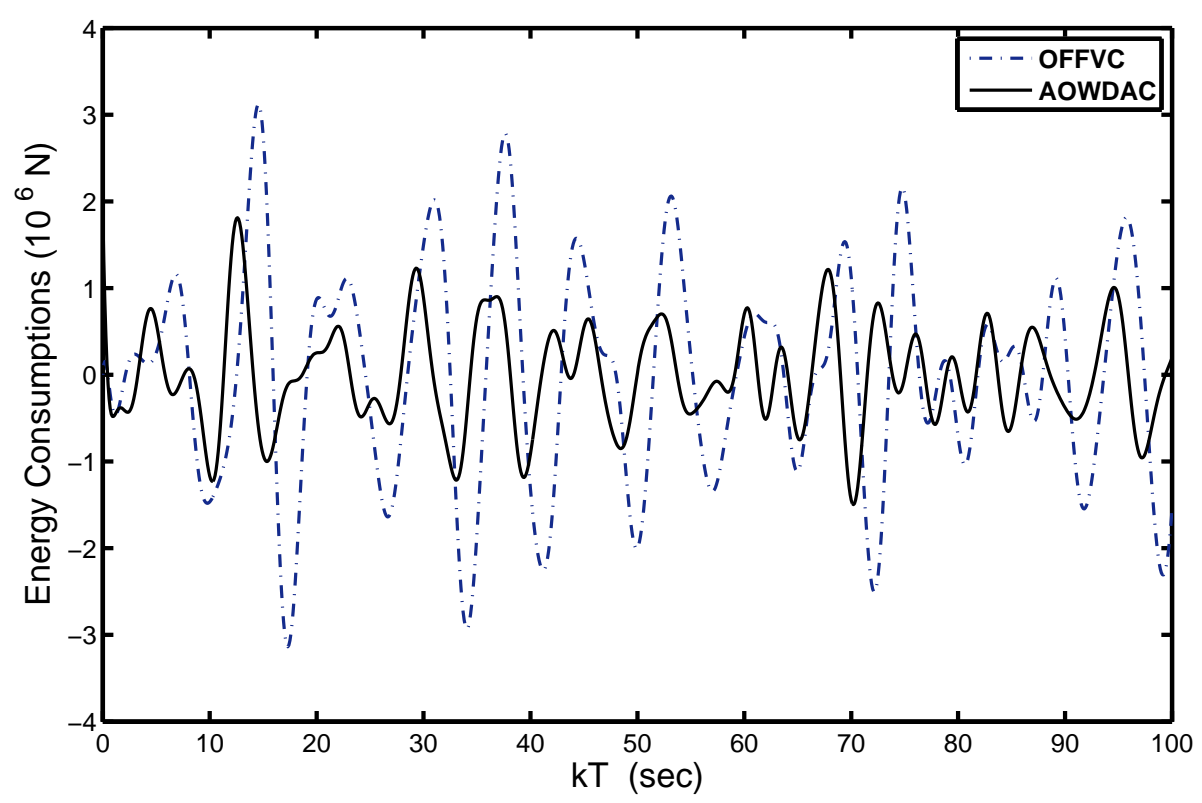

Figure 5. The curves of control forces of offshore platforms under different control schemes.

Table 3. Performance index values in each iteration.

\begin{tabular}{ccccc}
\hline Iteration Number & $\mathbf{1}$ & $\mathbf{2}$ & $\mathbf{3}$ & $\mathbf{4}$ \\
\hline Performance index $J$ & 4.7354 & 1.3662 & 0.9884 & 0.9792 \\
\hline
\end{tabular}

In order to show the wave disturbance attenuation abilities of proposed digital-control-based AOWDAC more clearly, the peak values and root-mean-square (RMS) values of the velocity, displacement and energy consumption of offshore platforms are listed in Tables 4 and 5 under the open-loop system (without control input), the proposed digital-control-based AOWDAC (33) and OFFVC (36).

Table 4. Peak values of the displacement, velocity and energy consumption, of offshore platforms under difference control schemes.

\begin{tabular}{cccc}
\hline Control Scheme & Displacement $(\mathbf{m})$ & Velocity $(\mathbf{m} / \mathbf{s})$ & $\left.\boldsymbol{u}(\mathbf{1 0})^{5}\right) \mathbf{N}$ \\
\hline Open-Loop & 0.3035 & 0.4468 & \\
Optimal feedback and feedfoward vibration controller (36) & 0.1914 & 0.2352 & 2.9925 \\
Approximation of optimal wave disturbances attenuation controller (33) & 0.1127 & 0.0910 & 1.6182 \\
\hline
\end{tabular}

Table 5. The root-mean-square values of the displacement, velocity and energy consumption, of offshore platforms under difference control schemes.

\begin{tabular}{cccc}
\hline Control Scheme & Displacement $(\mathbf{m})$ & Velocity $(\mathbf{m} / \mathbf{s})$ & $\boldsymbol{u}(\mathbf{1 0}) \mathbf{N}$ \\
\hline Open-Loop & 0.1206 & 0.1824 & \\
Optimal feedback and feedfoward vibration controller (36) & 0.0789 & 0.0902 & 1.1930 \\
Approximation of optimal wave disturbances attenuation controller (33) & 0.0451 & 0.0437 & 0.5884 \\
\hline
\end{tabular}

By analyzing Tables 3-5 and Figures 3-5, comparing OFFVC and digital-control-based AOWDAC, it can be summarized that the peak values and RMS values of the velocity and displacement of offshore platforms under digital-control-based AOWDAC are smaller than the ones under OFFVC. Meanwhile, the energy consumptions of digital-control-based AOWDAC are smaller than the ones under OFFVC. 
Therefore, the proposed digital-control-based AOWDAC can compensate the nonlinear dynamics of offshore platforms and attenuate the irregular wave disturbances effectively.

\section{Conclusions}

A digital-control-based AOWDAC was developed for a nonlinear jacket-type offshore platform under external irregular wave disturbances, which consists of feedback items of offshore platform state, feedfoward item for attenuating the external wave disturbance, and compensation sequences for responding to the nonlinear dynamic of offshore platforms. First, based on the JONSWAP wave spectrum and linearized wave theory, the wave forces were formulated as the output of an exosystem. Meanwhile, a discrete model of a nonlinear jacket-type offshore platform was established. After that, by solving an introduced nonlinear TPBV problem, the digital-control-based AOWDAC was designed based on the Riccati equation, Stein equation and two vector sequences. By designing an iterative algorithm, the feasibility of digital-control-based AOWDAC was realized based on the performance index in each iteration. Applying the digital-control-based AOWDAC to an offshore platform and comparing with OFFVC, the displacement and velocity of offshore platforms were reduced under proposed digital-control-based AOWDAC significantly, and the energy consumption of digital-control-based AOWDAC requires a smaller control force than other typical control scheme.

Due to the limitations of actuators, sensors and communication networks, one aspect of our future work will focus on the vibration control problem for the networked nonlinear offshore platform with delays and faults in actuator and measurements. Meanwhile, the predictive wave disturbance attenuation problem for offshore platforms is another aspect of our future work based on the wave feedforward information measured from advanced sensor technology.

Acknowledgments: This work was supported by the Natural Science Foundation of Shandong Province, China (Grant No. ZR2017MF044), the Project of Shandong Province Higher Educational Science and Technology Program, China (Grant No. J17KA047, J16LN07, J16LB06, J17KA055), the Shandong Distinguished Middle-aged and Young Scientist Encourage and Reward Foundation, China (Grant No. BS2014DX015, ZR2016FB14), the National Key Research and Development Plan, China (No. 2016YFC0106000), the Natural Science Foundation of China (Grant No. 61640218, 61671220, 61573166, 61673357), and the Shandong Province Key Research and Development Program, China (Grant No. 2016GGX101022).

Author Contributions: Xiao-Fang Zhong designed the control schemes; Yu-Hong Sun analyzed the data; Shi-Yuan Han wrote the paper; Jin Zhou and Dong Wang conceived and designed the experiments.

Conflicts of Interest: The authors declare no conflicts of interest.

\section{References}

1. Zhang, B.-L.; Han, Q.-L.; Zhang, X.-M. Recent advances in vibration control of offshore platforms. Nonlinear Dyn. 2017, 89, 755-771.

2. Yang, J.S. Hybrid active and passive control of a very large floating beam structure. Nonlinear Dyn. 2017, 87, 1835-1845.

3. Han, S.-Y.; Tang, G.-Y.; Chen, Y.-H.; Tang, X.-X.; Yang, X. Optimal vibration control for vehicle active suspension discrete-time systems with actuator time delay. Asian J. Control 2013, 15, 1579-1588.

4. Zhang, B.-L.; Han, Q.-L.; Zhang, X.-M. Robust non-fragile sampled-data control for offshore steel jacket platforms. Nonlinear Dyn. 2016, 83, 1939-1954.

5. Zhang, B.-L.; Ma, L.; Han, Q.-L. Sliding mode $H \infty$ control for offshore steel jacket platforms subject to nonlinear self-excited wave force and external disturbance. Nonlinear Anal. Real World Appl. 2013, 14, 163-178.

6. Zhang, B.-L.; Huang, Z.-W.; Han, Q.-L. Delayed non-fragile $H \infty$ control for offshore steel jacket platforms. J. Vib. Control 2015, 21, 959-974.

7. Wang, L.; Yang, B.; Chen, Y.; Zhang, X.; Orchard, J. Improving neural-network classifiers using nearest neighbor partitioning. IEEE Trans. Neural Netw. Learn. Syst. 2017, 28, 2255-2267.

8. Yu, Z.; Liu, Y.; Yu, X.; Pu, K.Q. Scalable distributed processing of K nearest neighbor queries over moving objects. IEEE Trans. Knowl. Data Eng. 2015, 27, 1383-1396. 
9. Kandasamy, R.; Cui, F.; Townsend, N. A review of vibration control methods for marine offshore structures. Ocean Eng. 2016, 127, 279-297.

10. Faltinsen, O.M. Sea Loads on Ships and Offshore Structures; Cambridge University Press: Cambridge, UK, 1990.

11. Kandasamy, R.; Cui, F.; Townsend, N. A systematic study of the lowest order small slope approximation for a Pierson-Moskowitz spectrum. IEEE Geosci. Remote Sens. Lett. 2011, 8, 158-162.

12. Annalisa, C.; Constance, M.S. Characterizing JONSWAP rogue waves and their statistics via inverse spectral data. Wave Motion 2017, 71, 5-17.

13. Cho, H.-Y.; Kweon, H.-M.; Jeong, W.-M.; Kim, S.-I. A study on the optimal equation of the continuous wave spectrum. Int. J. Nav. Archit. Ocean Eng. 2015, 7, 1056-1063.

14. Zhang, B.-L.; Liu, Y.-J.; Han, Q.-L.; Tang, G.-Y. Optimal tracking control with feedforward compensation for offshore jacket platforms with active mass damper mechanisms. J. Vib. Control 2016, 22, 695-709.

15. Zhang, B.-L.; Han, Q.-L. Network-based modelling and active control for offshore steel jacket platform with TMD mechanisms. J. Sound Vib. 2014, 333, 6796-6814.

16. Zhang, B.-L.; Han, Q.-L.; Zhang, X.-M. Event-triggered Ho reliable control for offshore structures in network environments. J. Sound Vib. 2016, 368, 1-21.

17. Yang, J.S. Robust mixed $\mathrm{H}_{2} / \mathrm{H} \infty$ active control for offshore steel jacket platform. Nonlinear Dyn. 2014, 2, 1503-1514.

18. Zhang, B.-L.; Han, Q.-L.; Zhang, X.-M.; Yu, X. Sliding mode control with mixed current and delayed states for offshore steel jacket platforms. IEEE Trans. Control Syst. Technol. 2014, 22, 1769-1783.

19. Nourisola, H.; Ahmadi, B. Robust adaptive sliding mode control based on wavelet kernel principal component for offshore steel jacket platforms subject to nonlinear wave-induced force. J. Vib. Control 2016, 22, 3299-3311.

20. Han, S.-Y.; Chen, Y.-H.; Tang, G.-Y. Sensor fault and delay tolerant control for networked control systems subject to external disturbances. Sensors 2017, 17, 700, doi:10.3390/s17040700.

21. Han, S.Y.; Zhang, C.-H.; Tang, G.-Y. Approximation optimal vibration for networked nonlinear vehicle active suspension with actuator time delay. Asian J. Control 2017, 19, 983-995.

22. Han, S.-Y.; Chen, Y.-H.; Tang, G.-Y. Fault diagnosis and fault-tolerant tracking control for discrete-time systems with faults and delays in actuator and measurement. J. Frankl. Inst. 2017, 354, 4719-4738.

23. Han, S.Y.; Wang, D.; Chen, Y.H.; Tang, G.Y.; Yang, X.X. Optimal tracking control for discrete-time systems with multiple input delays under sinusoidal disturbances. Int. J. Control Autom. Syst. 2015, 13, $292-301$.

24. Goebel, M.; Raitums, U. Constrained control of a nonlinear two point boundary value problem, I. J. Glob. Optim. 1994, 4, 367-395.

25. Chanane, B. Optimal control of nonlinear systems: A recursive approach. Comput. Math. Appl. 1998, 35, $29-33$.

26. Beard, R.W.; Saridis, G.N.; Wen, J.T. Galerkin approximation of the generalized Hamilton-Jacobi-Bellman equation. Automatica 1997, 33, 2159-2177.

27. Tang, G.-Y.; Gao, D.-X. Approximation design of optimal controllers for nonlienar systems with sinusoidal disturbances. Nonlinear Anal. Theory Methods Appl. 2007, 66, 403-414.

28. Ma, H.; Tang, G.-Y.; Zhao, Y.-D. Feedforward and feedback optimal control for offshore structures subjected to irregular wave forces. Ocean Eng. 2006, 33, 1105-1117.

(C) 2017 by the authors. Licensee MDPI, Basel, Switzerland. This article is an open access article distributed under the terms and conditions of the Creative Commons Attribution (CC BY) license (http://creativecommons.org/licenses/by/4.0/). 